\title{
A Probabilistic Representation of LiDAR Range Data for Efficient 3D Object Detection
}

\author{
Theodore C. Yapo ${ }^{1 *}$, Charles V. Stewart ${ }^{2}$, and Richard J. Radke ${ }^{1}$ \\ ${ }^{1}$ Department of Electrical, Computer, and Systems Engineering \\ ${ }^{2}$ Department of Computer Science \\ Rensselaer Polytechnic Institute, Troy, New York 12180 \\ yapot@rpi.edu, stewart@cs.rpi.edu, rjradke@ecse.rpi.edu
}

\begin{abstract}
We present a novel approach to $3 D$ object detection in scenes scanned by LiDAR sensors, based on a probabilistic representation of free, occupied, and hidden space that extends the concept of occupancy grids from robot mapping algorithms. This scene representation naturally handles LiDAR sampling issues, can be used to fuse multiple LiDAR data sets, and captures the inherent uncertainty of the data due to occlusions and clutter. Using this model, we formulate a hypothesis testing methodology to determine the probability that given $3 D$ objects are present in the scene. By propagating uncertainty in the original sample points, we are able to measure confidence in the detection results in a principled way. We demonstrate the approach in examples of detecting objects that are partially occluded by scene clutter such as camouflage netting.
\end{abstract}

\section{Introduction}

Light Detection and Ranging (LiDAR) scanners use time-of-flight measurements of narrow beams of laser light to produce estimates of the locations of 3D points in a scene. The resolution of commercially available LiDAR scanners can be very good, achieving an accuracy of a few $\mathrm{mm}$ at $100 \mathrm{~m}$ range [13]. However, unlike digital image sensors that use an optical low-pass filter to prevent the aliasing of high spatial frequencies in the scene, LiDAR sensors are very susceptible to sampling artifacts, as illustrated in Figure 1. For example, if the samples are too far apart, a LiDAR scan of a picket fence might be interpreted as a solid wall. Conversely, if a solid wall is sampled at a shallow grazing angle by nearly parallel LiDAR rays, it can be difficult to connect the distant sample points into a single sur-

\footnotetext{
* This work was supported in part by the US Army Intelligence and Security Command under the award W9124Q-04-F-2159, and by the DARPA Computer Science Study Group under the award HR0011-07-1-0016.
}

face. Hence, even though each range point is measured with high accuracy, there can still be quite a bit of uncertainty about the scene in each LiDAR scan.

Occlusions in the scene introduce a second source of uncertainty into LiDAR range data. Objects may be wholly or partially hidden from the point of view of the scanner, resulting in uncertainty about their presence or position in the scene. To deal with this issue effectively, a 3D object detection algorithm must allow fusion of data taken from different viewpoints, and model occlusion explicitly, noting what parts of the scene are visible from each viewpoint.

Much previous research on analyzing LiDAR data is based on generating a 3D model of the scene, either reducing the data to a polygonal model, or in some cases, producing an implicit function representation of the scene surfaces. Instead of irrevocably collapsing information about the scene into a likely "crisp" estimate, we propose to preserve the inherent uncertainty of the original data when testing hypotheses against the scene using a probabilistic framework.

We propose a discrete scene data structure to maintain a probabilistic model of the 3D scene, and provide a natural and tractable means to update this model that properly handles LiDAR sampling issues. The scene data structure is fundamentally a site occupancy probability model, extending the concept of occupancy grids from robotics [15]. We approximate the scene by a set of random fields that describe the probabilities that any single site (3D voxel) is in one of three states: free space, occupied, or hidden. This approach provides a sound basis for fusing data from disparate sensors that observe the scene from different viewpoints.

While we believe that the precision of available LiDAR sensors far surpasses that required for reliable object detection (since most objects of interest in outdoor scenes are very large relative to the uncertainty of a single LiDAR return), we cannot scan the scene with fewer LiDAR points without exacerbating the undersampling and aliasing prob- 


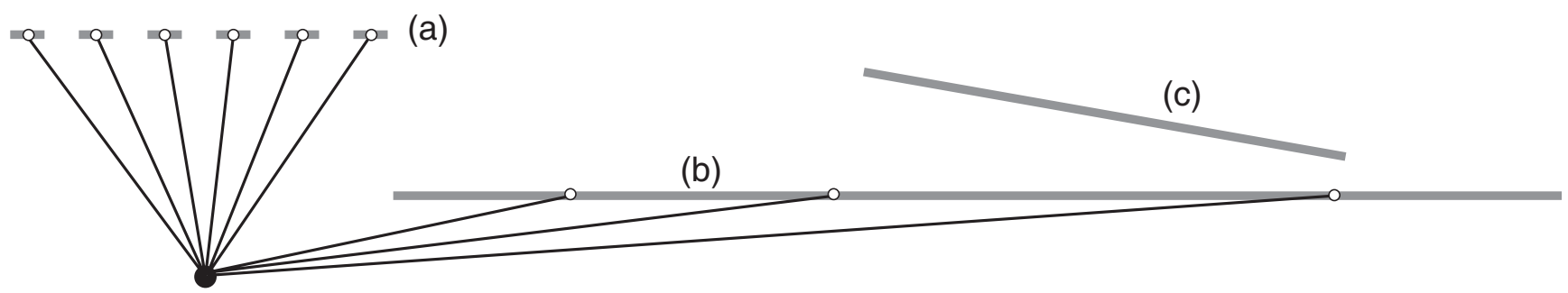

Figure 1. Illustrating sources of uncertainty in LiDAR data. The black dot represents the LiDAR sensor, the black lines LiDAR rays, the gray lines object surfaces, and the white dots LiDAR returns. LiDAR is susceptible to (a) undersampling of objects with holes, (b) poor sampling of surfaces due to shallow grazing angle, and (c) unsampled space resulting from occluding surfaces.

lems. To address these issues, we propose a downsampling technique to reduce data storage requirements and allow efficient hypothesis testing, while not glossing over important LiDAR sampling issues.

Our initial results are based on range data alone, but we note that our proposed data structure allows for the fusion of data from sensors of different modalities, such as digital images. Using our probabilistic model, 3D inferences from stereo pairs or single images could be used to "fill in" regions that are ambiguous or occluded from the viewpoint of the LiDAR scanner. Since image sensors typically have much higher resolution than their LiDAR counterparts, this combination could also be used to reduce uncertainty arising from LiDAR undersampling in the scene.

Finally, we describe how 3D hypothesis tests related to object detection can be easily performed on the data structure, using an efficient method based on the 3D Fast Fourier Transform. We demonstrate our results on an example where objects are hidden behind a camouflage net that makes the detection problem in raw data difficult.

\section{Related Work}

Some of the earliest models for representing range data used an octree with each leaf representing one of three unambiguous states: empty, occupied, or unseen [4]. Matthies and Elfes [15] initially proposed maintaining probabilities in a 2D structure called an occupancy grid for mapping the local environment of an autonomous robot. In the original approach, each cell in the grid was assigned a single value, $P^{o c c}$, representing the probability that the site was occupied; initial values for each cell were set to $\frac{1}{2}$ and the probabilities were updated using a Bayesian rule [16]. Subsequent methods [8, 19] assigned two evidence masses, one for empty $\left(m_{e}\right)$ and one for full $\left(m_{f}\right)$ such that $m_{e}+m_{f} \leq 1$ to capture some notion of uncertainty, and applied Dempster-Shafer theory [21] to update the grid.

Most approaches have treated the collection of cells as a Markov Random Field (MRF) of order 0 [5], i.e., assuming that the occupancy probabilities of neighboring cells are independent, greatly simplifying the occupancy probability estimation procedure. In contrast, Thrun [22] used a forward model of the sensor and an expectation-maximization algorithm to explicitly model the conditional dependencies of cells within a sensor sweep area. Jang et al. [10] clustered cells into sets using neural networks and modeled the conditional probabilities within these clusters. Ribo and Pinz [20] compared three types of models for updating the grid: probabilistic (using the Bayes update rule), DempsterShafer (using the Dempster rule of combination), and "possibilistic" (using fuzzy logic). Gorodnichy and Armstrong $[7,8]$ formulated the update rule as a regression. Matthies and Elfes [15] illustrated the importance of an update rule that is both commutative and associative, allowing data to be fused in any order.

Although much of the previous work on occupancy grids has been limited to 2D representations, several researchers have extended the notion to $3 \mathrm{D}$ scenes. One of the most important issues is avoiding a computationally intractable increase in the number of cells. Payeur et al. [19] described an octree representation in which spherical occupancy grids are computed around each sensor to accurately model the sensor characteristics; these are then merged into a common Cartesian grid. Several schemes for storing functional representations of occupancy fields have also been proposed. Payeur [18] described one such method in which a continuous spatial potential force is computed from the occupancy probabilities of each cell, allowing a continuous estimation of the field. Gorodnichy and Armstrong [8] proposed a parametric representation using a min-max tree of piecewise linear functions. Yguel et al. [24] proposed a 2D wavelet-based occupancy field, exploiting the continuous and natural multi-resolution properties of the wavelet decomposition. Paskin and Thrun [17] proposed using polygonal random fields for probabilistic mapping of 2D scenes, avoiding discretization errors and independence assumptions associated with occupancy grids. However, we note that most of these representations do not easily lend themselves to object recognition.

Once the data has been fused into a single model, hypotheses (e.g., related to the presence of objects) can be tested against it. One class of basic hypotheses concerns the 
detection or recognition of known 3D object models in the scene. Campbell and Flynn [3] give a good overview of the basic approaches to 3D object recognition. Among these, the most relevant to scenes containing heavy clutter and intentional occlusions (camouflage) are "point-feature" based methods. In these algorithms, a vector descriptor is calculated for a subset of points in the scene. These descriptors are then matched with those calculated from object models to detect and recognize objects in the scene. These methods can be robust to scene clutter and occlusions provided that initial local surface normals can be estimated.

Johnson and Hebert [11, 12] devised a point-feature based method known as "spin images". In this approach, a local surface normal is first estimated for each point in the scene to be tested. A 2D histogram is then rotated around this normal, creating a "spin image" representative of the local surface features of the object. The resulting image serves as a point descriptor which can then be matched with descriptors from object models.

Frome et al. [6] proposed two point-feature techniques and compared their performance to spin images. The first method extended the concept of shape contexts (Belongie et al. [1]) into 3D. In this method, a local surface normal is first estimated for each feature point. A spherical histogram of neighboring points is then calculated as the feature descriptor. A spherical harmonic transformation is then applied to this histogram to create a "harmonic shape context". Their results indicate better recognition rates than spin images.

Several researchers have applied these techniques to more real-world recognition problems. Vasile and Marino [23] developed an automatic target recognition algorithm for twelve vehicle classes in relatively-unoccluded LiDAR data based on spin images [12]. Huber et al. [9] described a parts-based classification algorithm for vehicles, applied to isolated recognition in the absence of clutter.

In these point-feature approaches, a local surface representation (either polygonal meshes or oriented points) is first estimated from the range points, discarding much of the uncertainty in the original data. For example, to generate and match spin images, one must accurately estimate surface normals at each 3D scene point to define spin axes. In contrast, our method assumes no a priori model of the scene or object structure, and was designed for datasets where millions of range points acquired from sensors at many different viewpoints are fused into a common frame. Real outdoor examples of this type of data include "fuzzy" regions like trees, large hidden regions, and sparsely-sampled areas arising from rays nearly parallel to object surfaces. Generating reliable surface and normal estimates in such cases would be difficult. However, as we describe below, these cases are naturally handled by occupancy modeling.

Our proposed object detection method uses FFT-based correlation to efficiently compute joint probabilities of in- dependent voxels. In this way, it is similar to FFT-based registration methods such as that presented by Lucchese et al. [14], which uses a correlation technique to estimate translational parameters in range data registration once rotational parameters have been estimated.

\section{Probabilistic Occupancy Model}

We assume the viewpoint for each LiDAR return is known; this is used to generate a model representing free and hidden space as well as regions occupied by solid objects. However, we do not require that the range points have a particular structure, such as lying on an angular grid; in fact, each range point can be taken from a different (known) viewpoint. Since each LiDAR point is considered independently, we treat estimation of occupancy probabilities as a sensor fusion problem even for data points obtained from a single sensor at a fixed position. This approach naturally scales to handle multiple LiDAR data sets that can be registered into a common world coordinate system.

Figure 2 illustrates our representation of both the scene space and objects that may be located within it. The scene space ideally comprises three disjoint sets: (1) the occupied points, which have been seen by one or more LiDAR sensors, (2) the hidden points, which have been obscured from all sensors, and (3) the free-space points, which have been "seen through" by at least one sensor. Also shown in Figure 2 is our representation of objects to be detected in the scene, as will be described in Section 4. Again, we consider three disjoint sets of points: (1) points on the object surface, which could possibly be seen by a LiDAR sensor, (2) points on the object's topological interior, which are always self-occluded by the object, and hence can never be seen, and (3) points exterior to the object, which may either be unoccupied, or contain part of a different object.

Using these models of the scene and object spaces, we can list the constraints that must be satisfied for an object to exist in the scene, as summarized in Table 1. The object's surface points must coincide with occupied scene points, and its interior points must coincide with hidden points. Conversely, points in an object's surface or interior cannot coincide with scene space known to be empty. Hidden space points may or may not be consistent with an object's surface being present; we address this issue in Section 4 . We finally note that scene points exterior to the object have no bearing on detection.

Our model for spatial occupancy of the scene is a set of random fields, in which we assign probabilities of being in each state independently to points in the scene, extending the ideas of [5]. For any point $\mathrm{x}$ in the scene space, we consider the probabilities that $\mathbf{x}$ is in one of the three states: occupied, hidden, or free, as illustrated in Figure 2:

$$
P^{o c c}(\mathbf{x})+P^{h i d}(\mathbf{x})+P^{f r e e}(\mathbf{x})=1 .
$$



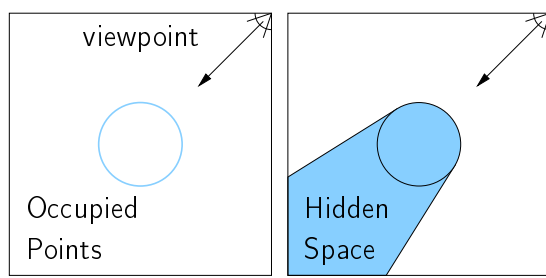

(a)

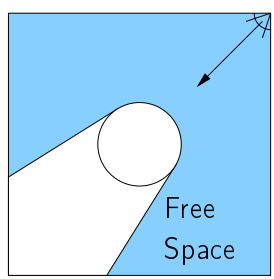

pace

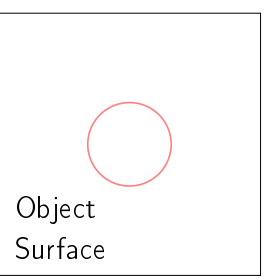

Interior
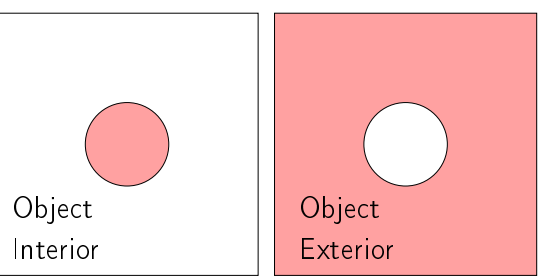

(b)

Figure 2. Six spaces of interest for constructing probability density functions of scene occupancy. (a) Representation of the scene. Each point in space is assumed to be in one of three states: occupied, hidden, or free. (b) Representation of an object. The object model is represented by three sets of points: surface, topological interior, and exterior.

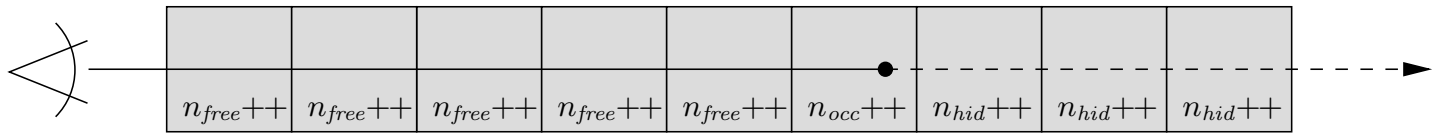

Figure 3. A single LiDAR return provides information about scene structure at every point along the view ray.

\begin{tabular}{|c|c|c|c|}
\hline & \multicolumn{3}{|c|}{ Scene Space } \\
\hline Object Space & occupied & hidden & free \\
\hline surface & $\sqrt{ }$ & $?$ & $\times$ \\
\hline interior & $\times$ & $\sqrt{ }$ & $\times$ \\
\hline exterior & - & - & - \\
\hline
\end{tabular}

Table 1. Constraints imposed by scene and object space states. " $\sqrt{ }$ " indicates a required coincidence, " $X$ " indicates mutual exclusivity, "?" indicates possible consistency, and "-" indicates no information gain.

We note the distinction between this model and previous work, which has either modeled site occupancy with a single probability [15] such that $P^{o c c}+P^{\text {free }}=1$, or with two evidence masses such that $m_{\text {occ }}+m_{\text {free }} \leq 1$ [8]. In our model, we consider the "hidden" state to be a distinct third possibility, not simply a lack of knowledge about the occupied or free state of the point, thereby accounting for points that are inside solid objects. In doing so, we allow a resolution to the problem of disambiguating unknown states from conflicting evidence [8]. For example, in single-probability models [15], unseen areas of space are assigned the noninformative prior $P^{o c c}=P^{\text {free }}=\frac{1}{2}$, but there is no way to distinguish this from a completely observed site where equal, but conflicting, evidence exists for either of the two states. Although Dempster-Shafer approaches [16] resolve this ambiguity by setting initial masses to $m_{o c c}=m_{\text {free }}=0$, they do not capture the important representation of hidden or occluded space. By making the third state explicit, we maintain true probabilities at each point, and can test hypotheses that the random field model is in a state consistent with the presence of an object in a principled way. Moreover, by maintaining a notion of hidden space, we enable more complex hypotheses involving the interior space of objects, as well as their sur- faces, to be tested. These tests are described in Section 4.

For each voxel in the grid, we wish to estimate the three probabilities, $P^{o c c}, P^{\text {free }}$, and $P^{\text {hid }}$. Since we assume the accuracy of each LiDAR point is much greater than the resolution of the voxel grid, we consider only the number of LiDAR returns that fall into each voxel for occupancy probability calculation. Likewise, for estimating $P^{\text {free }}$ and $P^{\text {hid }}$, we consider the number of view rays that pass through the voxel, or terminate in a LiDAR return before reaching it, respectively.

To efficiently accumulate these statistics, view rays are tracked through the grid using a variation of Bresenham's line drawing algorithm [2]. Each voxel is augmented with three counters: $n_{\text {free }}, n_{\text {occ }}$, and $n_{\text {hid }}$. Starting from the scanner position, each voxel in the path of the ray encountered before the LiDAR return has $n_{\text {free }}$ incremented. For the voxel containing the LiDAR return, $n_{\text {occ }}$ is incremented. The ray path is then continued until it leaves the grid, with each subsequent voxel having $n_{h i d}$ incremented. This process is illustrated in Figure 3.

Once all view rays have been traced through the grid, the three probabilities are estimated for each voxel. In doing so, we want completely unexplored voxels to be assigned the noninformative prior probabilities $P^{f r e e}=P^{o c c}=P^{\text {hid }}=$ $\frac{1}{3}$. As the number of points accumulated in the voxel increases, the collected statistics should begin to dominate the a priori estimates. This behavior would naturally be provided by a Bayesian update rule. However, in our case we have neither an accurate noise model for the scanner characteristics nor any knowledge about the scene structure within a voxel, so estimating the conditional probabilities required for a Bayesian rule is problematic. Instead, we estimate the voxel occupancy probability by:

$$
P^{o c c}=\frac{1}{3} \alpha^{n}+\left(1-\alpha^{n}\right)\left(\frac{n_{o c c}}{n}\right)
$$


for the given voxel, where $n=n_{\text {free }}+n_{\text {occ }}+n_{\text {hid }}$. The parameter $\alpha$ causes the influence of the a priori estimates to diminish exponentially with each additional ray traversing the voxel. The remaining two probabilities for each voxel, $P^{\text {hid }}$ and $P^{\text {free }}$, are estimated similarly using $n_{\text {hid }}$ and $n_{\text {free }}$, respectively.

\section{Hypothesis Testing}

Once the spatial probabilities at each voxel have been estimated, we can test the hypothesis that an object model as illustrated in Figure 2 is present in the scene, centered on a specific voxel. Instead of testing this hypothesis directly, we test the surrogate hypothesis that the random field model of the scene is in a state consistent with the presence of the object. The difference is subtle, as described below.

The object model comprises two sets of points, the set of points on the object surface:

$$
\mathbf{S}=\left\{\mathbf{x}_{i}^{S}, i=1, \ldots, N\right\}
$$

and the set of points topologically interior to the object:

$$
\mathbf{I}=\left\{\mathbf{x}_{j}^{I}, j=1, \ldots, M\right\},
$$

where $\mathbf{x}$ represents a point location in the object's coordinate system. We denote $\mathbf{S}_{\mathbf{x}_{0}}$ as the set of surface points translated so that their center is at point $\mathbf{x}_{0}$ :

$$
\mathbf{S}_{\mathbf{x}_{\mathbf{0}}}=\left\{\mathbf{x}_{i}^{S}+\mathbf{x}_{0}, i=1, \ldots, N\right\} .
$$

$\mathbf{I}_{\mathbf{x}_{0}}$ is defined similarly.

To determine the probability that a single voxel in the scene space is consistent with the presence of an object's surface, we could evaluate the probability that the point is occupied, $P^{o c c}(\mathbf{x})$, alone. However, doing so neglects the possible states of hidden points, which may either be hidden because they are truly part of an object's topological interior, or may simply have been occluded from all viewpoints in the LiDAR dataset. To account for this possibility, we apply a noninformative prior, assuming that hidden points can be either part of a surface or not with equal probability, since detection of intentionally hidden objects is particularly important in our application. We define the new probabilities that the voxel is consistent with the surface of an object or not:

$$
\begin{aligned}
P^{\text {surf }}(\mathbf{x}) & =P^{o c c}(\mathbf{x})+\frac{P^{h i d}(\mathbf{x})}{2} \\
P^{\neg \operatorname{surf}}(\mathbf{x}) & =P^{\text {free }}(\mathbf{x})+\frac{P^{h i d}(\mathbf{x})}{2}
\end{aligned}
$$

We note that these adjusted probabilities still obey $P^{\operatorname{surf}}(\mathbf{x})+P^{\neg \operatorname{surf}}(\mathbf{x})=1$. We take $P^{\operatorname{surf}}(\mathbf{x})$ to represent the probability that an object surface point is present at $\mathrm{x}$.
We now consider the probability $P\left(\mathbf{S}_{\mathbf{x}_{0}}\right)$ that the entire object surface $\mathbf{S}$ exists in the scene with the object origin at location $\mathbf{x}_{0}$. To do so, we calculate the probability that the random field model of the scene space is in a state consistent with this hypothesis:

$$
P\left(\mathbf{S}_{\mathbf{x}_{0}}\right)=\prod_{i=1}^{N} P^{\text {surf }}\left(\mathbf{x}_{i}^{S}+\mathbf{x}_{0}\right) .
$$

We similarly define $P\left(\mathbf{I}_{\mathbf{x}_{0}}\right)$, the probability that the object interior $\mathbf{I}$ is present in the scene centered at $\mathbf{x}_{0}$. Since the salient characteristic of object interior points is that they are always hidden from view (occluded by the object itself), we obtain:

$$
P\left(\mathbf{I}_{\mathbf{x}_{0}}\right)=\prod_{j=1}^{M} P^{h i d}\left(\mathbf{x}_{j}^{I}+\mathbf{x}_{0}\right)
$$

for the probability that the random field model is in a state consistent with the interior of the object being located at $x_{0}$.

Our ultimate goal is to express the probability $P^{o b j}\left(\mathbf{x}_{0}\right)$ that the object is present in the scene at location $\mathbf{x}_{0}$. For this to be the case, the random field must be in a state consistent with both the surface and the interior of the object being present centered at $\mathbf{x}_{0}$ :

$$
\begin{aligned}
P^{o b j}\left(\mathbf{x}_{0}\right) & =P\left(\mathbf{S}_{\mathbf{x}_{0}}\right) P\left(\mathbf{I}_{\mathbf{x}_{0}}\right) \\
& =\prod_{i=1}^{N} P^{s u r f}\left(\mathbf{x}_{i}^{S}+\mathbf{x}_{0}\right) \prod_{j=1}^{M} P^{h i d}\left(\mathbf{x}_{j}^{I}+\mathbf{x}_{0}\right) .
\end{aligned}
$$

Since we have modeled spatial occupancy with a random field for which occupancy probabilities are independent, calculation of these probabilities is straightforward. To facilitate efficient calculation and avoid numerical issues, we transform the product of (10) to a sum by considering the log likelihood:

$$
\begin{aligned}
\ln P^{o b j}\left(\mathbf{x}_{0}\right)= & \sum_{i=1}^{N} \ln P^{s u r f}\left(\mathbf{x}_{i}^{S}+\mathbf{x}_{0}\right) \\
& +\sum_{j=1}^{M} \ln P^{h i d}\left(\mathbf{x}_{j}^{I}+\mathbf{x}_{0}\right) .
\end{aligned}
$$

The sums of (11) can more easily be interpreted by a change of notation, in which we explicitly index voxels and object models by a triple index:

$$
\begin{aligned}
P_{i, j, k}^{o b j}=\exp \left(\sum_{l=1}^{N_{1}} \sum_{m=1}^{N_{2}} \sum_{n=1}^{N_{3}} S_{l, m, n} \ln P_{i+l, j+m, k+n}^{\text {surf }}\right. \\
\left.+\sum_{l=1}^{M_{1}} \sum_{m=1}^{M_{2}} \sum_{n=1}^{M_{3}} I_{l, m, n} \ln P_{i+l, j+m, k+n}^{h i d}\right) \\
=\exp \left(S \star \ln P^{s u r f}+I \star \ln P^{h i d}\right),
\end{aligned}
$$


where $P_{i, j, k}^{o b j}$ is the probability that the random field model is in a state consistent with the object being centered at voxel $(i, j, k), S_{i, j, k}$ is the discretized object surface model, $I_{i, j, k}$ is the discretized object interior model, $P_{i, j, k}$ is the appropriate discretized spatial probability, and $\star$ denotes crosscorrelation. In this form, (12) is easily recognized as the sum of two 3D cross correlations of the object surface and interior models with the log probabilities of surface and hidden states of the random field model, respectively.

Calculation of the full cross correlations of (12) can be achieved in $O\left(N^{3} \lg N\right)$ time for a 3D grid of $N$ voxels per side using a Fast Fourier Transform (FFT) algorithm. Since this computation simultaneously calculates the probability of the object existing in the scene centered at each of the $N^{3}$ voxel sites, the cost per hypothesis test is $O(\lg N)$. In practice, further savings can be realized when testing multiple objects against the same scene, since the computationally intensive step of transforming the log spatial probabilities into the Fourier domain only needs to be done once.

Moreover, the computation required for hypothesis testing is not a function of the number of LiDAR returns or scans used; this only affects the pre-processing time, which is linear in the number of points. Once the scene probabilities are constructed, the object detection time is not a function of the number of points used to estimate the spatial probabilities, nor is it a function of object complexity; the only parameter affecting the detection time is the number of voxels in the grid.

\section{Results and Discussion}

The proposed method was applied to an example scene consisting of a cylinder of $0.26 \mathrm{~m}$ radius and a box of $0.41 \mathrm{~m}$ per side obscured behind camouflage netting, as shown in Figure $4 \mathrm{a}$. The scene was scanned with a Leica HDS LiDAR scanner that collected 130,299 range points with an approximate depth accuracy of $2 \mathrm{~mm}$. We present the processing of two horizontal slices of the full 3D scan to facilitate visualization. As shown in Figure 4b, one slice intersects the cylinder, while the second slice intersects the box; the cross-sections of the objects become a circle and a square, respectively. A slice consists of $400 \times 400$ voxels, each measuring $12 \mathrm{~mm}$ on a side.

The parameter $\alpha$ used in estimating the voxel probabilities of (2) controls the rate at which the a priori probabilities decay as LiDAR rays are added to the scene. In practice, we found a value of $\alpha=0.9$ to work well, which was used in obtaining the results of Figure 4. The mean number of LiDAR points in each non-empty voxel was 3.2 , while the maximum number in any voxel was 26.

Figure 4c illustrates a map of the random field model of the first scene slice, with $P^{\text {free }}, P^{o c c}$, and $P^{\text {hid }}$ mapped to the red, green, and blue channels, respectively. Hence, completely unseen voxels which have equal a priori prob- abilities appear as a mid gray. Figure $4 d$ illustrates similar maps for the second slice containing the box. As can be seen in the figures, the camouflage netting occludes most of each object from the LiDAR viewpoint (i.e., virtually all points behind the net have $P^{h i d} \approx 1$ ).

We formed object models consisting of the cylinder at its true size and two boxes smaller than the true size, as illustrated in Figure 4e. Testing the cylinder model against the voxel slice intersecting the cylinder produces the detection probability map of Figure 4f. In this figure, the true center of the cylinder is clearly identified, with much smaller probability that the cylinder is centered on nearby voxels, and nearly zero chance that a cylinder is present elsewhere in the scene.

In Figure 4g, the same test is repeated for the case of the $0.38 \mathrm{~m}$ box model. In this case, we see that since the box model is slightly smaller than the actual box in the scene, there is some correctly-estimated ambiguity about where the box could exist in the scene. The smaller box model "slides" within the boundaries of the scanned box's front edge, since the sides of the box are almost entirely occluded from the single LiDAR viewpoint. These occlusions produce a natural uncertainty in the result, which could be resolved by scanning the scene from an additional viewpoint. This effect is even more pronounced when the smaller box model $(0.19 \mathrm{~m}$ on a side $)$ is tested against the same scene (Figure $4 \mathrm{~h}$ ). In this case, the random field model is consistent with placement of the smaller box anywhere along a short line of positions, with the greatest probability near the middle of the front box surface.

We note that it would be difficult to directly compare these results against spin images or 3D shape contexts since we do not assume we know or can find local surface normals, which are required for either method. In many scenes of interest, where objects are intentionally hidden, for example, by camouflage netting, estimating reliable surface normals will be problematic. Additionally, our data structure has been designed so that images can be fused with $\mathrm{Li}$ DAR range scans to fill in occluded regions or compensate for LiDAR undersampling. Our detection algorithm could be directly applied to such fused data. However, spin images and 3D shape contexts are not naturally extended to leverage additional information from images.

\section{Conclusions and Future Work}

Our framework enables probabilistic 3D object detection using a spatial occupancy model derived from LiDAR range data. Within this framework, range points from multiple LiDAR scans can be fused into a common representation that captures uncertainty in the scene caused by occlusion and sampling artifacts. This uncertainty can then be meaningfully propagated to the object detection results.

We believe there are a number of areas for further re- 


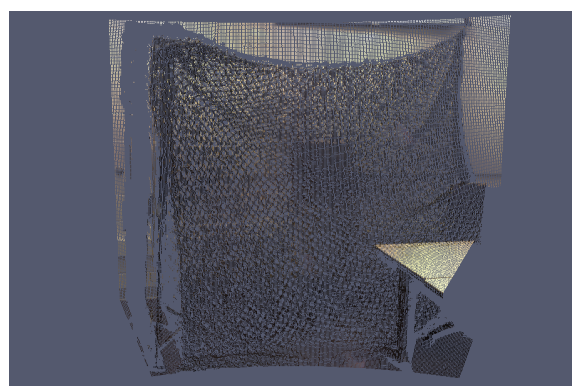

(a)

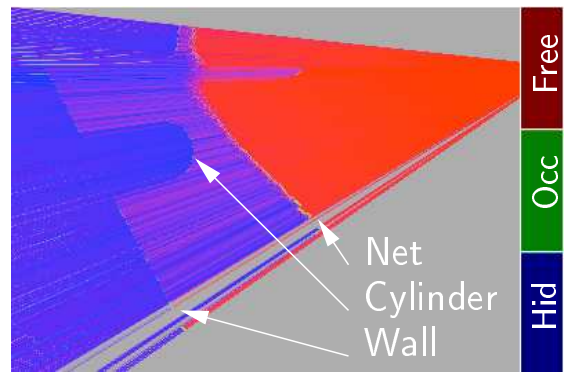

(c)

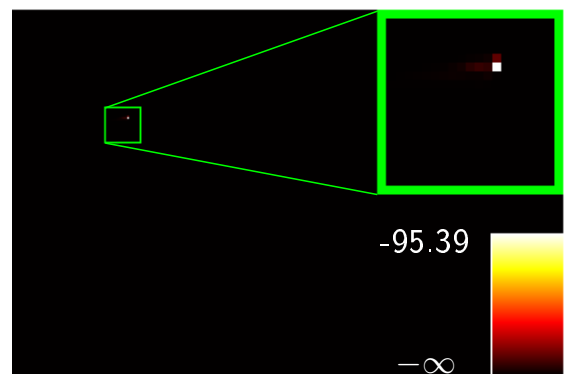

(f)

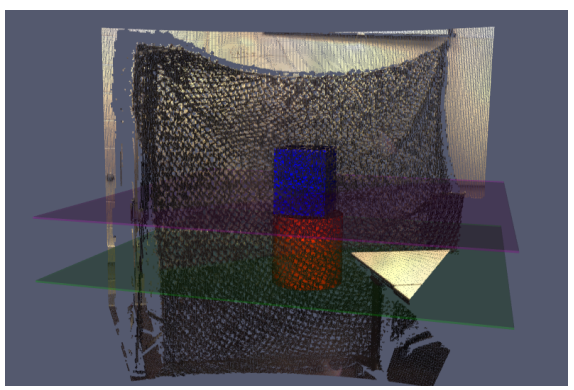

(b)

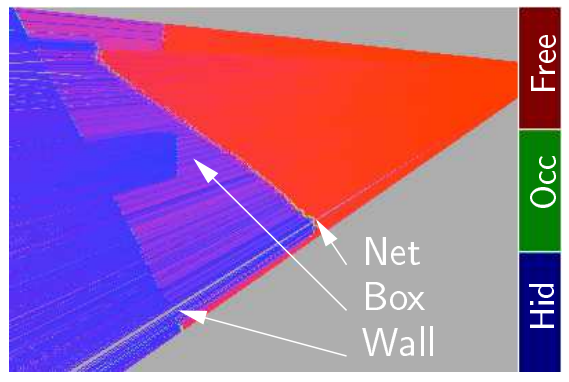

(d)

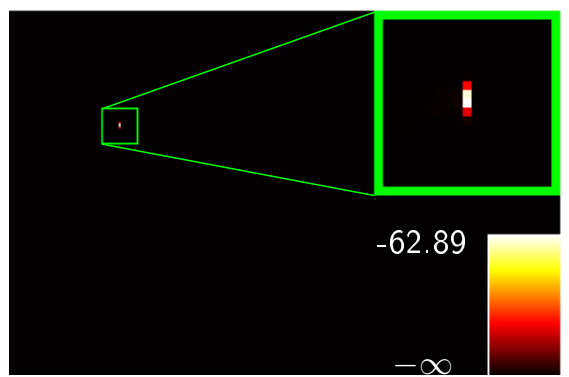

(g)

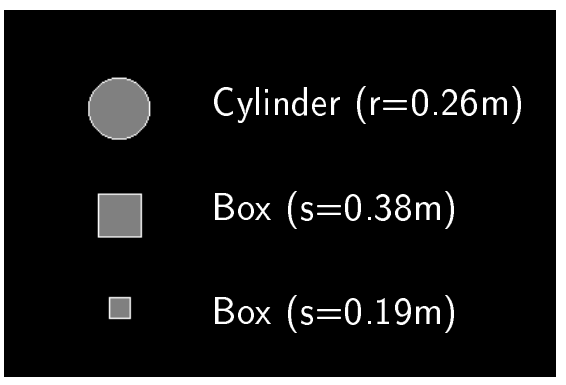

(e)

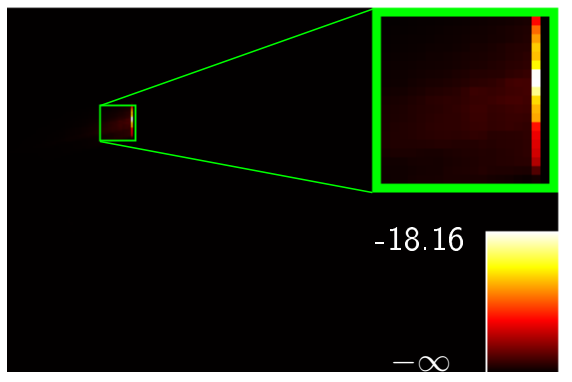

(h)

Figure 4. Probabilistic detection of objects partially occluded by camouflage netting. (a) LiDAR data of the scene. (b) LiDAR data showing manually-inserted true location of cylinder (red) and box (blue). (c) Probabilistic occupancy map of green horizontal slice of (b). Free probabilities are mapped to the red channel, hidden probabilities to the blue channel, and occupied probabilities to the green channel. (d) Probabilistic occupancy map of purple horizontal slice of (b). (e) Object models (horizontal slices). (f) The calculated log probability of the presence of the cylindrical model at each voxel in the scene. (g) Calculated probability of presence of large box model. (h) Calculated probability of presence of small box model.

search extending our scene representation and object detection framework. While the current formulation of the technique provides no explicit rotational invariance, many object models are invariant to some degree. This natural invariance can be exploited in a naive search over pose parameters, made feasible using the fast FFT-based algorithm. We are currently investigating more elegant and efficient methods for imparting full rotational invariance to the method.

Although the independent random field model of spatial occupancy facilitates efficient computation, it implies some counter-intuitive assumptions about the scene structure, e.g., that the probability of an object's interior and its surface being collocated are independent. We plan to investigate higher-order MRF models of spatial occupancy that include conditional probabilities between neighboring cells. While this approach may yield more accurate models and more manageable probabilities, the main challenge will be maintaining computational efficiency, since this will preclude the use of the fast FFT-based algorithm in its current form. It remains to be seen whether detection results would be improved.

Accurate estimation of spatial occupancy is critical to the success of the proposed technique. The current method uses an implicit spatial "box" filter to downsample the LiDAR returns. While this greatly mitigates sampling issues, it is not a perfect anti-aliasing filter. Ideally, we should filter the LiDAR data with a "box" filter in the frequency domain that removes all frequencies above the voxel Nyquist rate. Since the LiDAR points are relatively sparse, this could be efficiently accomplished with a straightforward $\sin (x) / x$ inter- 
polation.

Although using a logarithmic representation avoids numerical issues involving the small magnitudes of the detection probabilities, these magnitudes currently depend on the cardinality of the points in the object model. Hence, while we can straightforwardly interpret the detection results for a single object, comparing results derived from object models of widely different sizes is problematic. We are investigating the normalization of the results relative to the object model size so that different detection maps can be directly compared.

Finally, we note that if multiple objects are to be tested against a single scene, the linearity of the cross correlation operations can be exploited to improve efficiency. If the collection of object models can be decomposed into a common set of primitive objects, these primitives can be tested against the scene, and the detection results combined in the logarithmic representation of (11) to produce detection results for the composite objects.

\section{References}

[1] S. Belongie, J. Malik, and J. Puzicha. Shape matching and object recognition using shape contexts. IEEE Trans. Pattern Anal. Mach. Intell., 24(4):509-522, 2002.

[2] V. Boyer and J.-J. Bourdin. A faster algorithm for 3D discrete lines. In Proceedings of the Euorpean Association for Computer Graphics Conference, 1998.

[3] R. J. Campbell and P. J. Flynn. A survey of free-form object representation and recognition techniques. Comput. Vis. Image Underst., 81(2):166-210, 2001.

[4] C. Connolly. Cumulative generation of octree models from range data. In Proceedings of the IEEE International Conference on Robotics and Automation, pages 25-32, 1984.

[5] A. Elfes. Using occupancy grids for mobile robot perception and navigation. IEEE Computer, 22(6):46-57, 1989.

[6] A. Frome, D. Huber, R. Kolluri, T. Bulow, and J. Malik. Recognizing objects in range data using regional point descriptors. In Proceedings of the European Conference on Computer Vision, 2004.

[7] D. Gorodnichy. On using regression in range data fusion. In Proceedings of the Canadian Conference on Electrical and Computer Engineering (CCECE'99), May 1999.

[8] D. Gorodnichy and W. Armstrong. A parametric alternative to grids for occupancy-based world modeling. In Proceedings of Quality Control by Artificial Vision (QCAV'99), May 1999.

[9] D. Huber, A. Kapuria, R. Donamukkala, and M. Hebert. Parts-based 3D object classification. In Proceedings of the IEEE Conference on Computer Vision and Pattern Recognition, June 2004.

[10] B. Jang, T. Choi, and J. Lee. Adaptive occupancy grid mapping with clusters. Artificial Life and Robotics, 10:162165(4), November 2006.
[11] A. Johnson. Spin-Images: A Representation for 3-D Surface Matching. PhD thesis, Robotics Institute, Carnegie Mellon University, Pittsburgh, PA, August 1997.

[12] A. Johnson and M. Hebert. Using spin images for efficient object recognition in cluttered 3D scenes. IEEE Transactions on Pattern Analysis and Machine Intelligence, 21(5):433449, May 1999.

[13] T. Kremen, B. Koska, and J. Pospil. Verification of laser scanning systems quality. In XXIII International FIG Congress, 2006.

[14] L. Lucchese, G. Doretto, and G. Cortelazzo. A frequency domain technique for range data registration. IEEE Transactions on Pattern Analysis and Machine Intelligence, 24(11):1468-1484, Nov 2002.

[15] L. Matthies and A. Elfes. Integration of sonar and stereo range data using a grid-based representation. In Proceedings of the IEEE International Conference on Robotics and Automation, volume 2, April 1988.

[16] D. Pagac, E. Nebot, and H. Durrant-Whyte. An evidential approach to probabilistic map-building. In Proceedings of the IEEE International Conference on Robotics and Automation, volume 1, pages 745-750, 1996.

[17] M. A. Paskin and S. Thrun. Robotic mapping with polygonal random fields. In Proceedings of the 21st Conference on Uncertainty in Artificial Intelligence, July 2005.

[18] P. Payeur. Improving robot path planning efficiency with probabilistic virtual environment models. In Proceedings of the IEEE Symposium on Virtual Environments, HumanComputer Interfaces and Measurement Systems, pages 1318, 2004.

[19] P. Payeur, P. Hebert, D. Laurendeau, and C. Gosselin. Probabilistic octree modeling of a 3D dynamic environment. In Proceedings of the IEEE International Conference on Robotics and Automation, pages 1289-1296, 1997.

[20] M. Ribo and A. Pinz. A comparison of three uncertainty calculi for building sonar-based occupancy grids. Int. J. Robotics and Autonomous Systems, 35:201-209, 2001.

[21] G. Shafer. A Mathematical Theory of Evidence. Princeton University Press, 1976.

[22] S. Thrun. Learning occupancy grids with forward models. In Proceedings of the IEEE/RSJ International Conference on Intelligent Robots and Systems, volume 3, pages 1676-1681, 2001.

[23] A. N. Vasile and R. M. Marino. Pose-independent automatic target detection and recognition using 3D laser radar imagery. Lincoln Laboratory Journal, 15(1), 2005.

[24] M. Yguel, O. Aycard, and C. Laugier. Wavelet occupancy grids: a method for compact map building. In Proc. of the Int. Conf. on Field and Service Robotics, 2005. 